Check for updates

Cite this: RSC Adv., 2019, 9, 17366

\title{
Facile fabrication of amphiphobic surfaces on copper substrates with a mixed modified solution $\uparrow$
}

\author{
Ning Wang, Qing Wang, (D) * Shuangshuang Xu, Xu Zheng and Mingya Zhang
}

Fabricating amphiphobic surfaces is often complex, difficult to control, and time-consuming, making the fabrication process very difficult. Herein, a facile and time-saving modification method using a mixed modified solution of stearic acid and perfluorooctanoic acid was initially proposed, which played a key role in the achievement of the superhydrophobicity and highly oleophobicity. The effects of reaction time on surface morphology and wettability as well as the content of perfluorooctanoic acid in the mixed modified solution on wettability were investigated to determine the optimal experiment parameters that maximized the amphiphobicity of the surfaces. The as-fabricated amphiphobic surfaces displayed high oil contact angles of $133.5^{\circ}$, higher water contact angles of $156.8^{\circ}$, ultra-low water sliding angles of less than $5^{\circ}$ and excellent self-cleaning properties. The facile, easy to control, and efficient method can provide new insights into fabricating amphiphobic surfaces and can open up a new way for the basic research and practical application of amphiphobic surfaces.

Received 10th April 2019

Accepted 28th May 2019

DOI: 10.1039/c9ra02688f

rsc.li/rsc-advances

surface energy. Guo et al..$^{22}$ created copper mesh with superhydrophobic and oleophobic properties via etching samples in a mixed acid solution of $\mathrm{HCl}$ and $\mathrm{CH}_{3} \mathrm{COOH}$ for $24 \mathrm{~h}$, modification with a $1 H, 1 H, 2 H, 2 H$-perfluorooctyltriethoxysilane solution for up to $12 \mathrm{~h}$, a self-assembled monolayer can be formed under special acid-base reaction conditions, and then the hightemperature experimental conditions were set to heat the samples in an oven for $2 \mathrm{~h}$ to achieve the purpose of reducing the surface energy. Lee et $a l .{ }^{23}$ fabricated non-wettable surface through etching copper in an ammonium ambient solution of $\mathrm{NaOH}$ and $\left(\mathrm{NH}_{4}\right)_{2} \mathrm{~S}_{2} \mathrm{O}_{8}$, heating at $180{ }^{\circ} \mathrm{C}$ for $60 \mathrm{~min}$, and modifying with $1 H, 1 H, 2 H, 2 H$-perfluorooctyl under vacuum for $2 \mathrm{~h}$, and only by using special vacuum experimental conditions, the low surface energy can be achieved. These fabricating methods which required special experimental conditions and equipment added the difficulty of fabrication and control of experimental conditions. Moreover, modifying the surface with a low-surface energy material was time consuming, which limited the large-scale production of amphiphobic surfaces. To overcome these problems, it has become an urgent need to propose a facile, easy to control, and efficient method for fabricating amphiphobic surfaces on copper substrates with a mixed modified solution.

The lowest known surface energy materials can be arranged in descending order as $\mathrm{CH}>\mathrm{CH}_{2}>\mathrm{CH}_{3}>\mathrm{CF}_{2}>\mathrm{CF}_{3} \cdot{ }^{24,25}$ Stearic acid (STA, $\left.\mathrm{CH}_{3}\left(\mathrm{CH}_{2}\right)_{16} \mathrm{COOH}\right)$ with $16-\mathrm{CH}_{2}$ groups and $1-\mathrm{CH}_{3}$ group is a low surface energy material, but it only possesses hydrophobicity and not oleophobicity. Perfluorooctanoic acid $\left(\mathrm{PFOA}, \mathrm{CF}_{3}\left(\mathrm{CF}_{2}\right)_{6} \mathrm{COOH}\right)$ has $6-\mathrm{CF}_{2}$ groups and $1-\mathrm{CF}_{3}$ group, wherein a $-\mathrm{CF}_{3}$ group has the lowest surface energy of $6.7 \mathrm{mN}$ $\mathrm{m}^{-1} \cdot{ }^{26}$ Hence, $\mathrm{PFOA}$ is hydrophobic and oleophobic with a quite

Institute of NanoEngineering, College of Civil Engineering and Architecture, Shandong University of Science and Technology, Qingdao 266590, China.E-mail: qwang@sdust. edu.cn

† Electronic supplementary information (ESI) available. See DOI: 10.1039/c9ra02688f 
lower surface energy. In addition, the reactive group - $\mathrm{COOH}$ in both STA and PFOA can react with metals and their compounds to bond low surface energy groups to the surface to achieve the purpose of reducing the surface energy. ${ }^{27,28}$ It can be confirmed that a mixed modified solution composed of STA and PFOA can improve the hydrophobicity due to the STA and achieve the oleophobicity due to the PFOA. Thereby, mixing STA and PFOA at a reasonable ratio to form a mixed modified solution can shorten the modification time to obtain amphiphobic surfaces.

To the best of our knowledge, the method of modifying the surface using a mixture of two low surface energy materials to fabricate amphiphobic surfaces on copper substrates has not been reported. In this study, we created micro-nano hierarchical dendritic structures by immersing copper substrates into a silver nitrate aqueous solution and proposed a facile and timesaving modification method using a mixed modified solution of STA and PFOA for reducing surface energy to fabricate amphiphobic surfaces on copper substrates. The effects of reaction time on surface morphology and wettability as well as the content of PFOA in the mixed modified solution on wettability were investigated to determine the optimal experiment parameters that maximized the amphiphobicity of the surfaces. Furthermore, the self-cleaning property of the as-fabricated amphiphobic surfaces was also studied. The method designed here was facile, easy to control, and efficient, which can provide new insights into fabricating amphiphobic surfaces with a mixture of two low surface energy materials and can open up a new way for the basic research and practical application of amphiphobic surfaces.

\section{Experimental}

\section{Materials and reagents}

Copper substrates (purity $\geq 99.9 \%, 20 \mathrm{~mm} \times 30 \mathrm{~mm} \times 1 \mathrm{~mm}$ ) were provided by Shenzhen Zhibao Metal Products Co., Ltd., China. Silver nitrate $\left(\mathrm{AgNO}_{3}\right)$ was purchased from Sinopharm Group Chemical Reagent Co., Ltd., China. Stearic acid (STA, $\left.\mathrm{CH}_{3}\left(\mathrm{CH}_{2}\right)_{16} \mathrm{COOH}\right)$ was obtained from Tianjin Beilian Fine Chemicals Development Co., Ltd., China. Perfluorooctanoic acid (PFOA, $\mathrm{CF}_{3}\left(\mathrm{CF}_{2}\right)_{6} \mathrm{COOH}$ ) was provided by Shanghai Macklin Biochemical Technology Co., Ltd., China. Anhydrous ethanol was purchased from Tianjin Kemiou Chemical Reagent Co., Ltd., China. Blend oil was obtained from Luhua Co., Ltd., China. All chemical reagents were of analytical grade and used without further purification.

\section{Sample fabrication}

The copper substrate was polished with sandpapers to $2000 \#$ to remove the oxide layer on the substrate and cleaned ultrasonically with anhydrous ethanol and deionized water for $10 \mathrm{~min}$ respectively to remove surface grease. Subsequently, the copper substrate was immersed into $0.02 \mathrm{~mol} \mathrm{~L}^{-1} \mathrm{AgNO}_{3}$ aqueous solution at room temperature (about $26{ }^{\circ} \mathrm{C}$ ) for different times $(5,10,15$ and $20 \mathrm{~min})$. Afterwards, the substrate was rinsed with deionized water to eliminate any residual impurity and subsequently dried. Then, the substrate was modified by a mixed ethanol solution of STA and PFOA for $1 \mathrm{~h}$, and the total concentration of the two materials was maintained at $0.02 \mathrm{~mol} \mathrm{~L}^{-1}$. Finally, the substrate was removed, washed with ethanol and deionized water successively, and dried at room temperature.

Morphology and composition. The surface morphology of the samples was determined by a scanning electron microscope (SEM, APERO, FEI Co., Ltd. USA), equipped with an energydispersive X-ray spectroscopy (EDS) system for elemental analysis. The crystal structure of the samples was examined by X-ray diffraction (XRD, Rigaku Utima IV, Rigaku Co., Ltd., Japan). The chemical composition was recorded with a Fourier transform infrared (FT-IR) spectrometer (Nicolet 380 FT-IR, Thermo Electron Co., Ltd. USA).

Wettability measurements. Water contact angle (WCA) and oil contact angle (OCA) were measured on an optical contact angle measuring device (KRUSS-DSA30, Crass Scientific Instrument Co., Ltd., Germany) using distilled water and blend oil, respectively. Water sliding angle (WSA) was measured by a home-made experimental device composed by a backing plate (marked as a), two glass sheets (marked as b and c), a protractor (marked as d) and a triangle plate (marked as e) as showed in Fig. S1. $\uparrow$ The backing plate was placed horizontally on experiment table. Then the protractor was placed vertically along one side of the backing plate which upper side is flush with the $0^{\circ}$ scale line of the protractor. At the same time, a triangle plate was also placed behind the protractor to ensure that the protractor is perpendicular to the backing plate. Next placed two glass sheets on the backing plate, one is fixed on the plate (b), the other (c) can rotate along the baseline on backing plate corresponded by $90^{\circ}$ scale line of protractor. When measuring the sliding angle of the droplets, slowly lift the outside of the glass sheet (c) and record the position of the glass sheet when the droplets beginning to roll. At this time, the scale line corresponding to the glass sheet is the measured water sliding angle. All angles were measured with droplets of approximately $10 \mu \mathrm{L}$ at three different locations of the samples, and the average values were used for the subsequent data analysis.

Self-cleaning experiments. The self-cleaning property of the amphiphobic surface was tested. Firstly, a layer of white chalk powder as a contaminant was spread on the surface. Afterwards, water droplets of approximately $10 \mu \mathrm{L}$ were dropped onto the surface. Finally, the surface was cleaned by the rolling off of the water droplets.

\section{Results and discussion}

\section{Surface morphology}

The surface morphologies under different magnifications obtained when the polished $\mathrm{Cu}$ substrate was immersed into an $\mathrm{AgNO}_{3}$ aqueous solution for different times are exhibited in Fig. 1. The morphology of the bare copper substrate prior to $\mathrm{AgNO}_{3}$ immersion is shown in Fig. S2, $\uparrow$ from which only scratches on the surface can be observed, which is different from those after being immersed into an $\mathrm{AgNO}_{3}$ aqueous solution. As shown in Fig. 1a-d, the surface morphologies were mainly composed of dendritic structures. Fig. 1e-h exhibit that 

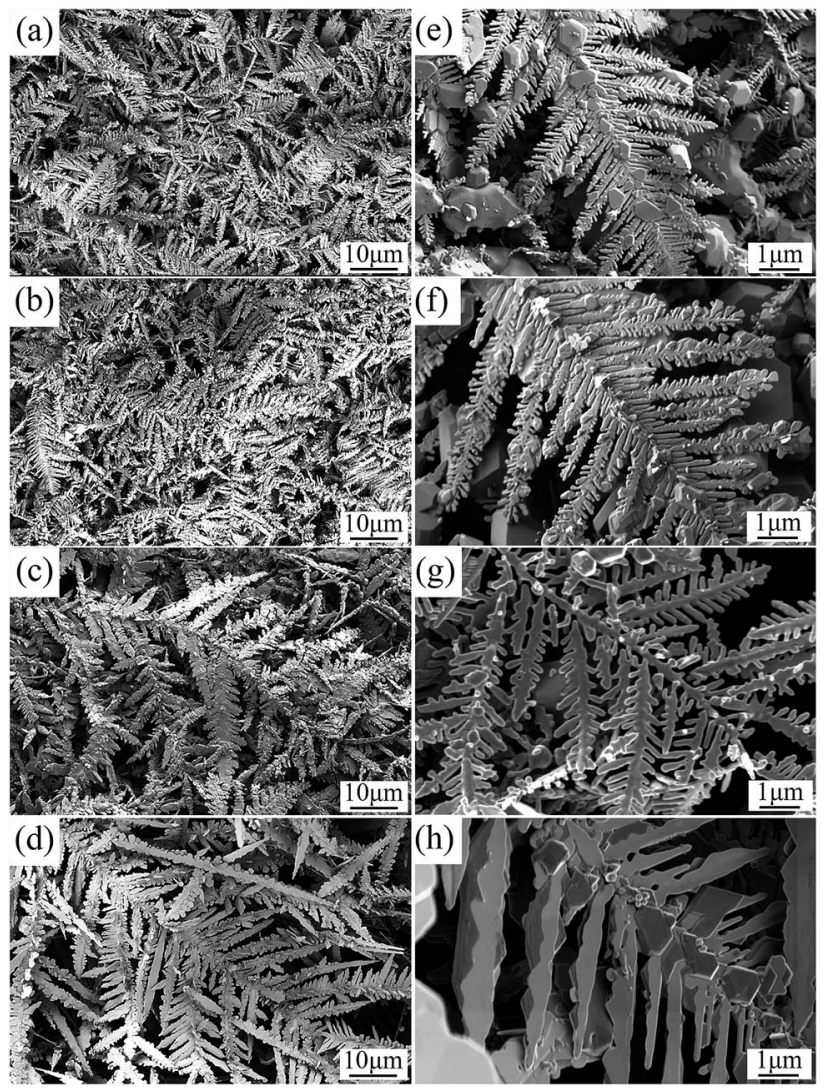

Fig. 1 SEM images of polished Cu substrate at different reaction times in an aqueous solution of $\mathrm{AgNO}_{3}$ under different magnifications: (a) and (e) $5 \mathrm{~min}$, (b) and (f) $10 \mathrm{~min}$, (c) and (g) $15 \mathrm{~min}$, (d) and (h) $20 \mathrm{~min}$.

under high magnification the feature structures of the surface morphologies mainly included micro-nano hierarchical dendritic structures with the width in the order of hundreds of nanometers and lengths in the order of microns of the main branches, and the width and length in the order of hundreds of nanometers of the small dendrites, growing from the main branches. Besides, there were a small number of irregular multi-sided cubic crystal structures (Fig. 1e-h).

When the immersion time was short (5 $\mathrm{min})$, as shown in Fig. 1a and e, the size of the dendritic structures was small. As the immersion time increased, the dendritic structures grew continuously and the size became larger. Extending the time to 15 min (Fig. 1c and g), micro-nano hierarchical dendritic structures were clearly visible, and the size significantly increased; therefore, the surface roughness greatly improved. When the time was increased to $20 \mathrm{~min}$ (Fig. 1d and h), the micro-nano hierarchical dendritic structures had changed. Although the size of the micro-scale structures still increased, the size of the nano-scale structures gradually decreased. The micro-nano hierarchical dendritic structures became insignificant hierarchical dendritic structures dominated by the microscale, which made the surface roughness decrease. It was concluded that when the immersion time was $15 \mathrm{~min}$, micronano hierarchical dendritic structures were significant and the surface morphology was the roughest. The large water-air interface area can effectively prevent water or oil droplets from penetrating the surface and form stable Cassie state. ${ }^{29}$

\section{Surface chemistry}

The chemical composition of the surfaces under different conditions was characterized by XRD, EDS and FT-IR. Fig. 2 presents the XRD patterns of the polished $\mathrm{Cu}$ substrate and $\mathrm{Ag} @ \mathrm{Cu}$ surface (after the polished $\mathrm{Cu}$ substrate was immersed into the $\mathrm{AgNO}_{3}$ aqueous solution). As shown in Fig. 2a, the polished $\mathrm{Cu}$ substrate exhibited diffraction peaks of $\mathrm{Cu}$ (111), $\mathrm{Cu}(200)$ and $\mathrm{Cu}(220)$ at $43.4^{\circ}, 50.53^{\circ}$ and $74.16^{\circ},{ }^{30}$ respectively. Except for the three diffraction peaks of the polished $\mathrm{Cu}$ substrate, four diffraction peaks of Ag (111), $\mathrm{Ag}$ (200), $\mathrm{Ag}$ (220) and $\mathrm{Ag}$ (311) appeared at $38.18^{\circ}, 44.38^{\circ}, 64.53^{\circ}$ and $77.48^{\circ}$ in the XRD pattern of the Ag@Cu surface (Fig. 2b), ${ }^{30,31}$ respectively. This implied that the polished $\mathrm{Cu}$ substrate was successfully covered with a silver film after being immersed into the $\mathrm{AgNO}_{3}$ aqueous solution. In addition, the diffraction peaks at $29.48^{\circ}$, $36.34^{\circ}, 42.39^{\circ}$, and $61.43^{\circ}$ were attributed to $\mathrm{Cu}_{2} \mathrm{O}(110), \mathrm{Cu}_{2} \mathrm{O}$ (111), $\mathrm{Cu}_{2} \mathrm{O}(200)$ and $\mathrm{Cu}_{2} \mathrm{O}(220),{ }^{30,32}$ which were the reason for the irregular multi-sided cubic crystal structures of the surface morphology (Fig. 1). We concluded that both $\mathrm{Ag}$ and $\mathrm{Cu}_{2} \mathrm{O}$ were formed on the polished $\mathrm{Cu}$ substrate after being immersed into the $\mathrm{AgNO}_{3}$ aqueous solution. The related reaction between $\mathrm{Cu}$ substrate and the $\mathrm{AgNO}_{3}$ aqueous solution can be further explained by eqn (1) and (2).33

$$
\begin{gathered}
\mathrm{Cu}(\mathrm{sol})+\mathrm{Ag}^{+}(\mathrm{aq}) \rightarrow \mathrm{Cu}^{2+}(\mathrm{aq})+\mathrm{Ag}(\mathrm{sol}) \\
\mathrm{Cu}(\mathrm{sol})+\mathrm{Cu}^{2+}(\mathrm{aq})+\mathrm{H}_{2} \mathrm{O}(\text { liq }) \rightarrow \mathrm{Cu}_{2} \mathrm{O}(\mathrm{sol})+2 \mathrm{H}^{+}(\mathrm{aq})
\end{gathered}
$$

EDS analysis was carried out to study the chemical composition of the polished $\mathrm{Cu}$ substrate, $\mathrm{Ag} @ \mathrm{Cu}$ surface, STA@Ag@Cu surface (after the Ag@Cu surface was modified by

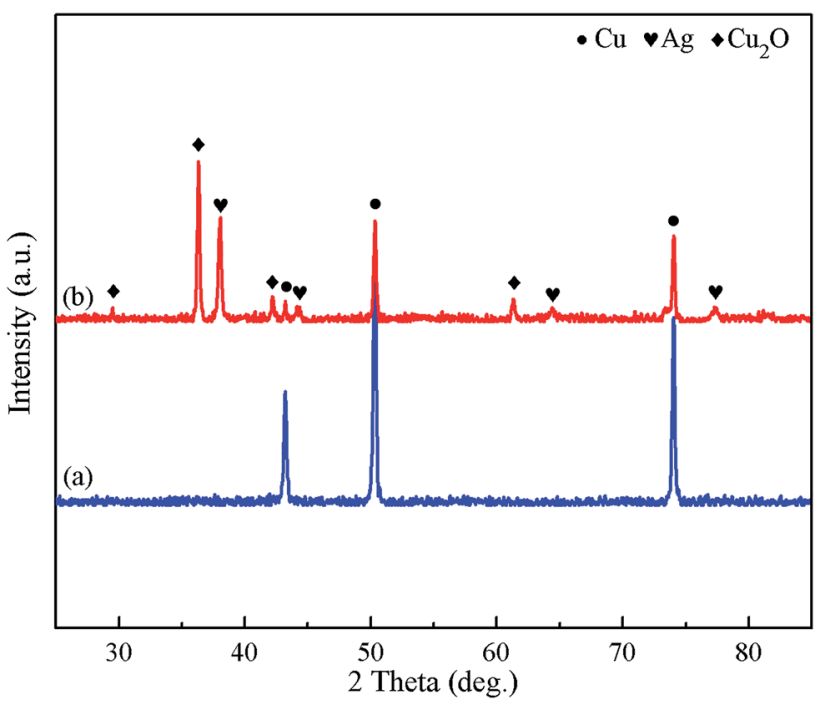

Fig. 2 XRD patterns of (a) polished $\mathrm{Cu}$ substrate and (b) $\mathrm{Ag} \mathrm{ACu}$ surface. 
STA), PFOA@Ag@Cu surface (after the Ag@Cu surface was modified by PFOA) and STA\&PFOA@Ag@Cu surface (after the $\mathrm{Ag} @ \mathrm{Cu}$ surface was modified by a mixture of STA and PFOA). As shown in Fig. S3a, $\uparrow$ the EDS spectrum of the polished $\mathrm{Cu}$ substrate contained only elemental $\mathrm{Cu}$. The elements $\mathrm{Ag}$ and $\mathrm{O}$ were observed in the EDS spectra of the Ag@Cu surface (Fig. S3b $\dagger$ ). Combined with Fig. 2b, it was confirmed that the silver film covered the polished $\mathrm{Cu}$ substrate and the elemental $\mathrm{O}$ was from $\mathrm{Cu}_{2} \mathrm{O}$. Related to Fig. $\mathrm{S} 3 \mathrm{~b}$, $\dagger$ elemental $\mathrm{C}$ appeared in the EDS spectrum of the STA@Ag@Cu surface (Fig. S3c $\dagger$ ), which demonstrated that the $\mathrm{Ag} @ \mathrm{Cu}$ surface may be successfully modified by STA. The elements $\mathrm{C}$ and $\mathrm{F}$ in Fig. S3d $\dagger$ were from PFOA, which indicated that the $\mathrm{Ag@Cu}$ surface may be successfully modified by PFOA. Fig. S3e $\dagger$ exhibits the elements $\mathrm{C}$ and $\mathrm{F}$, which showed that the mixture of STA and PFOA may successfully modify the Ag@Cu surface. This conclusion was further verified by the following FT-IR spectra test.

To further confirm whether the surface was successfully modified, FT-IR spectra were obtained, and are shown in Fig. S4. $\dagger$ After being modified by STA, the adsorption peaks at approximately $2918 \mathrm{~cm}^{-1}$ and $2848 \mathrm{~cm}^{-1}$ were assigned to $-\mathrm{CH}_{3}$ and $-\mathrm{CH}_{2}$ stretching vibrations, ${ }^{34,35}$ respectively (Fig. S4a $\dagger$ ). In combination with Fig. S3c, $\dagger$ it was believed that the STA@Ag@Cu surface had STA. After being modified by PFOA, the bands at approximately $1206 \mathrm{~cm}^{-1}$ and $1149 \mathrm{~cm}^{-1}$ were attributed to the stretching vibrations of the $-\mathrm{CF}_{2}$ and $-\mathrm{CF}_{3}$ groups (Fig. S4b $\dagger$ ). ${ }^{36}$ In combination with Fig. S3d, $\dagger$ PFOA was shown to be successfully bound to Ag@Cu surface. After being modified by the mixture of STA and PFOA, not only $-\mathrm{CH}_{2}$ and $-\mathrm{CH}_{3}$ groups, but also $-\mathrm{CF}_{2}$ and $-\mathrm{CF}_{3}$ groups were observed in Fig. S4c. $\dagger$ In combination with Fig. S3e, $\dagger$ we can confirm that the STA\&PFOA@Ag@Cu surface had STA and PFOA. Furthermore, the peaks at approximately $1744 \mathrm{~cm}^{-1}$ and $1640 \mathrm{~cm}^{-1}$ arose from the stretching vibration of the coordinated COOgroups. ${ }^{37}$ In addition, a broad band at approximately $3500 \mathrm{~cm}^{-1}$ arose from an $-\mathrm{OH}$ stretching vibration. ${ }^{38}$

\section{Surface wettability}

The wettability of droplets on a solid surface depends mainly on two factors: surface morphology and surface chemistry. ${ }^{39}$ The effects of reaction time and the PFOA content on the wettability of different surfaces were investigated. The molar ratio of PFOA in the mixed modified solution was expressed as $M_{\mathrm{F}}$. The wettability of the amphiphobic surface was evaluated by water and blend oil. Besides, the repellency to daily droplets such as tea and milk was also explored.

Effect of reaction time on amphiphobicity. The polished $\mathrm{Cu}$ substrates were immersed into an $\mathrm{AgNO}_{3}$ aqueous solution for different times $(5,10,15$ and $20 \mathrm{~min})$, and further modified for $1 \mathrm{~h}$ to determine the effect of the reaction time on the CA and SA for STA@Ag@Cu $\left(M_{\mathrm{F}}=0 \%\right), \mathrm{PFOA} @ \mathrm{Ag} @ \mathrm{Cu}\left(M_{\mathrm{F}}=100 \%\right)$ and 4 intermediate mixtures surfaces $\left(M_{\mathrm{F}}=20 \%, 40 \%, 60 \%, 80 \%\right)$.

The effect of the reaction time on the CA for STA@Ag@Cu, PFOA@Ag@Cu and 4 intermediate mixtures surfaces is shown in Fig. 3 and 4 . It was observed that the WCA were all greater than $150^{\circ}$ (Fig. 3), while the OCA varied in the range of about $50^{\circ}$

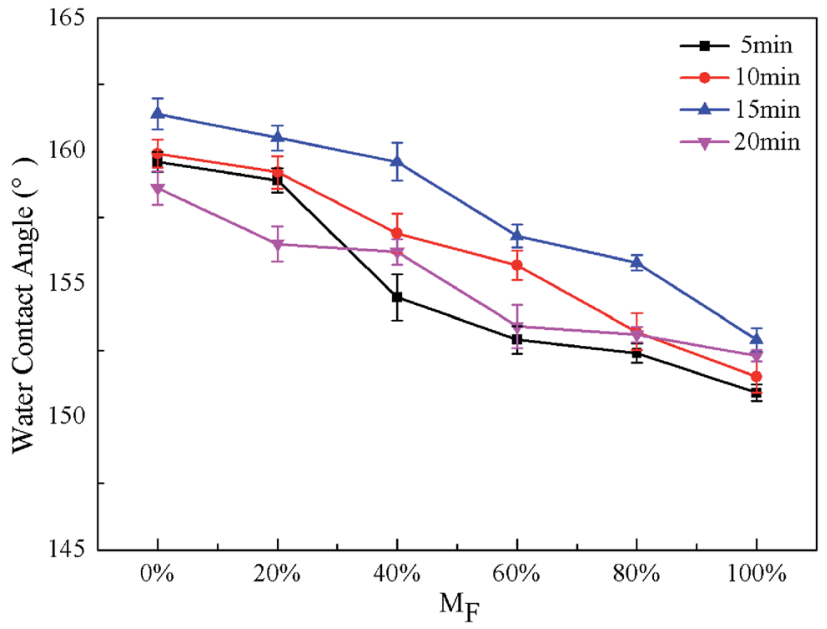

Fig. 3 Effects of reaction time and $M_{F}$ on WCA for STA@Ag@Cu $\left(M_{F}=\right.$ $0 \%)$, PFOA@AgaCu $\left(M_{\mathrm{F}}=100 \%\right)$ and 4 intermediate mixtures surfaces $\left(M_{F}=20 \%, 40 \%, 60 \%, 80 \%\right)$.

to $140^{\circ}$ at different reaction times (Fig. 4). Both WCA and OCA showed an upward trend with the increase of reaction time from 5 to 15 min under the same PFOA content. The WCA increased from $159.6^{\circ}$ to $161.4^{\circ}\left(M_{\mathrm{F}}=0 \%\right), 158.9^{\circ}$ to $160.5^{\circ}\left(M_{\mathrm{F}}=20 \%\right)$, $154.5^{\circ}$ to $159.6^{\circ}\left(M_{\mathrm{F}}=40 \%\right), 152.9^{\circ}$ to $156.8^{\circ}\left(M_{\mathrm{F}}=60 \%\right), 152.4^{\circ}$ to $155.8^{\circ}\left(M_{\mathrm{F}}=80 \%\right), 150.9^{\circ}$ to $152.9^{\circ}\left(M_{\mathrm{F}}=100 \%\right)$, respectively, and the range was within $5^{\circ}$. The OCA increased from $49.8^{\circ}$ to $52.8^{\circ}\left(M_{\mathrm{F}}=0 \%\right)$, from $53.1^{\circ}$ to $110.6^{\circ}\left(M_{\mathrm{F}}=20 \%\right)$, from $117.4^{\circ}$ to $124.7^{\circ}\left(M_{\mathrm{F}}=40 \%\right)$, from $118.2^{\circ}$ to $133.5^{\circ}\left(M_{\mathrm{F}}=60 \%\right)$, from $134.4^{\circ}$ to $137.2^{\circ}\left(M_{\mathrm{F}}=80 \%\right)$, and from $141.9^{\circ}$ to $145.5^{\circ}\left(M_{\mathrm{F}}\right.$ $=100 \%$ ), respectively, and the range was around $5-70^{\circ}$ (see Table $\mathrm{S} 1 \dagger)$. This indicated that the reaction time had a greater impact on the OCA than the WCA. Besides, the WCA was larger than the OCA at each time point, because the surface tension of oil is much smaller than that of water. ${ }^{\mathbf{1}}$ However, there was a certain downward trend at $20 \mathrm{~min}$. This indicated that the CA

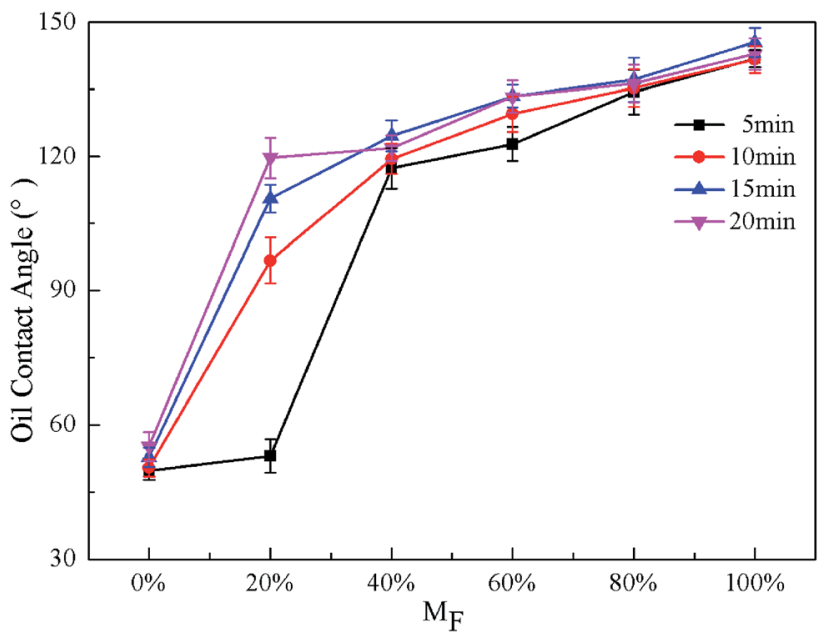

Fig. 4 Effects of reaction time and $M_{F}$ on OCA for STA@Ag@Cu $\left(M_{F}=\right.$ $0 \%)$, PFOA@Ag@Cu $\left(M_{\mathrm{F}}=100 \%\right)$ and 4 intermediate mixtures surfaces $\left(M_{\mathrm{F}}=20 \%, 40 \%, 60 \%, 80 \%\right)$. 
achieved the maximum when the reaction time was $15 \mathrm{~min}$, at which time a large amount of air was trapped in the gaps generated in the hierarchical dendritic structures. This was in keeping with the analysis of the surface morphology presented in Section 3.1.

The effect of the reaction time on WSA for STA@Ag@Cu, PFOA@Ag@Cu and 4 intermediate mixtures surfaces can be observed from Fig. 5. Except $20 \mathrm{~min}$, the WSA were all less than $5^{\circ}$ when the $M_{\mathrm{F}}$ was less than or equal to $60 \%$, in addition, WCA were all larger than $150^{\circ}$ (Fig. 3), indicating that the surfaces reached superhydrophobicity. Nevertheless, when $M_{\mathrm{F}}$ was more than $60 \%$, WSA suddenly increased. Once $M_{\mathrm{F}}$ reached $100 \%$, water droplets adhered to the surfaces, and WSA was recorded as $180^{\circ}$. For $20 \mathrm{~min}$, WSA was less than $10^{\circ}$ only when $M_{\mathrm{F}}$ was $0 \%$, while water droplets adhered to the surfaces when $M_{\mathrm{F}}$ was more than $0 \%$. The greater adhesion attributed to proper surface morphologies was not present (Fig. 1d and h).

Effect of PFOA content on the CA and SA. The effect of PFOA content on the CA and SA for STA@Ag@Cu,PFOA@Ag@Cu and 4 intermediate mixtures surfaces as explored through modified the Ag@Cu surface with different mixed modified solutions for $1 \mathrm{~h}$.

Fig. 3 and 4 also exhibit the effect of PFOA content on the CA. The WCA were all larger than $150^{\circ}$ under the same reaction time with the increase of $M_{\mathrm{F}}$, presenting a downward trend, whereas the OCA showed an upward trend, making the surfaces change from superoleophilic to oleophobic (Fig. 4). Except $5 \mathrm{~min}$, the OCA was larger than $90^{\circ}$ when the $M_{\mathrm{F}}$ was more than or equal to $20 \%$, showing that the surfaces reached oleophobicity and when the $M_{\mathrm{F}}$ was more than or equal to $60 \%$, the OCA exceeded $130^{\circ}$, displaying that the oleophobicity improved. When the reaction time was the same and the PFOA content increased from 0 to $100 \%$, the WCA decreased from $159.6^{\circ}$ to $150.9^{\circ}$ (5 $\min ), 159.9^{\circ}$ to $151.5^{\circ}(10 \mathrm{~min}), 161.4^{\circ}$ to $152.9^{\circ}(15 \mathrm{~min}), 158.6^{\circ}$ to $152.3^{\circ}$ (20 $\mathrm{min}$ ), respectively, and the range was within $10^{\circ}$.

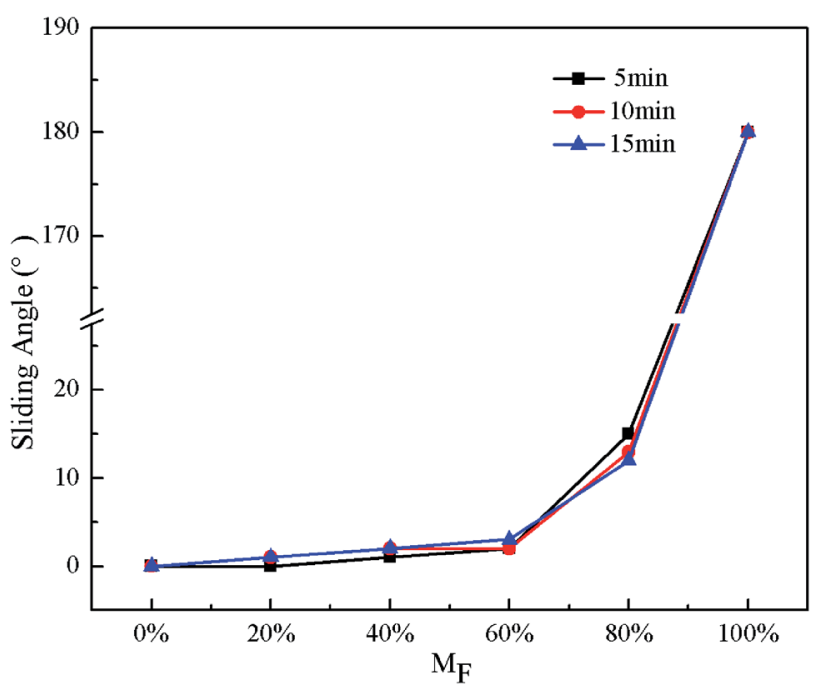

Fig. 5 Effects of reaction time and $M_{F}$ on WSA for STA@Ag@Cu $\left(M_{F}=\right.$ $0 \%)$, PFOA@Ag@Cu $\left(M_{F}=100 \%\right)$ and 4 intermediate mixtures surfaces $\left(M_{F}=20 \%, 40 \%, 60 \%, 80 \%\right)$.
The OCA increased from $49.8^{\circ}$ to $141.9^{\circ}(5 \mathrm{~min}), 50.4^{\circ}$ to $141.7^{\circ}$ $(10 \mathrm{~min})$, and $52.8^{\circ}$ to $145.5^{\circ}(15 \mathrm{~min}), 55.2^{\circ}$ to $148.7^{\circ}(20 \mathrm{~min})$, respectively, the range of variation reached around $90^{\circ}$ (see Table S $\left.{ }^{\dagger}\right)$. This revealed that the PFOA content made greater influence on OCA than WCA. The reason was that with the $M_{\mathrm{F}}$ increased, under the same reaction times more oleophobic groups $-\mathrm{CF}_{3}$ and $-\mathrm{CF}_{2}$ can be bonded to the surface, so the oleophobicity improved continuously. In addition, when the content of PFOA was the same and the reaction time increased from 5 to $15 \mathrm{~min}$, the range of the OCA was around $5-70^{\circ}$. While when the reaction time was the same and the content of PFOA ranged from 0 to $100 \%$, the range of the OCA reached around $90^{\circ}$. It can be inferred that the influence of PFOA content on the OCA was greater than the reaction time. Therefore, the amphiphobicity mainly depended on the PFOA content in the mixed modified solution.

Wettability of the amphiphobic surface. From the analysis of Sections 3.3.1 and 3.3.2, under the same PFOA content, when the reaction time was $15 \mathrm{~min}$, the WCA reached the maximum, the WSA was smaller, and the OCA was larger. Hence 15 min can be determined as the optimal reaction time parameter. The optimal parameter of the PFOA content at 15 min was further investigated. Fig. 6 demonstrates the relationship between the CA and PFOA content as well as the corresponding optical images of the CA at $15 \mathrm{~min}$. With the increase of $M_{\mathrm{F}}$, the WCA decreased slowly but were all larger than $150^{\circ}$, while the OCA increased continuously. When the $M_{\mathrm{F}}$ was $60 \%$, the OCA exceeded $130^{\circ}$ and the WSA was about $3^{\circ}$ (Fig. 7 and Video S1 $\dagger$ ). While once the $M_{\mathrm{F}}$ was greater than $60 \%$, WSA suddenly increased to be greater than $10^{\circ}$ (Fig. 5). As a result, the $M_{\mathrm{F}}$ of $60 \%$ was determined as the optimal parameter.

Consequently, amphiphobic surfaces with a high OCA of $133.5^{\circ}$ (Fig. 6), a higher WCA of $156.8^{\circ}$ (Fig. 6) and a small WSA of $3^{\circ}$ (Fig. 7), possessed superhydrophobicity and oleophobicity were fabricated when the reaction time was $15 \mathrm{~min}$ and the

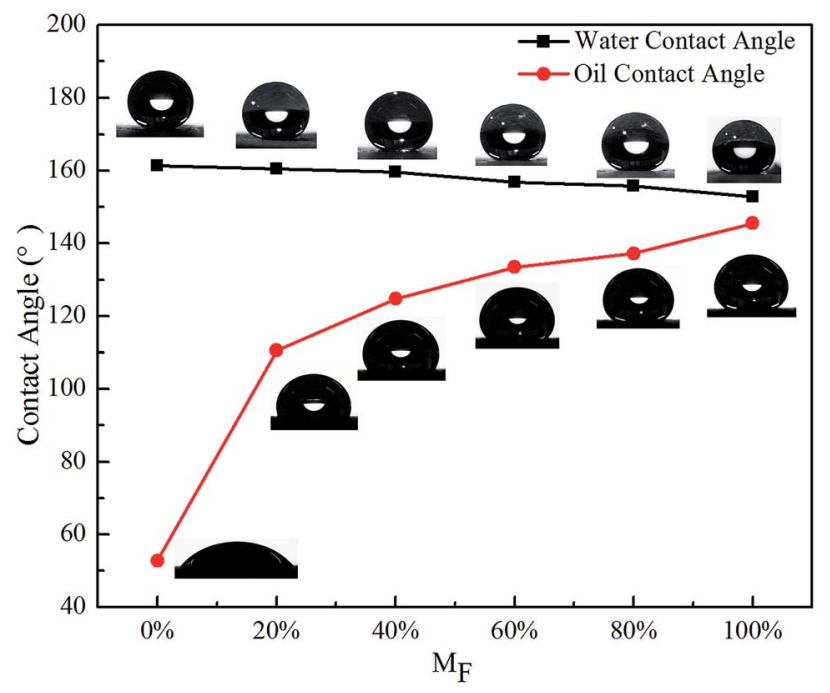

Fig. 6 Variation of CA with $M_{F}$ at 15 min reaction time for STA@Ag@Cu $\left(M_{\mathrm{F}}=0 \%\right)$, PFOA@Ag@Cu $\left(M_{\mathrm{F}}=100 \%\right)$ and 4 intermediate mixtures surfaces $\left(M_{F}=20 \%, 40 \%, 60 \%, 80 \%\right)$. 


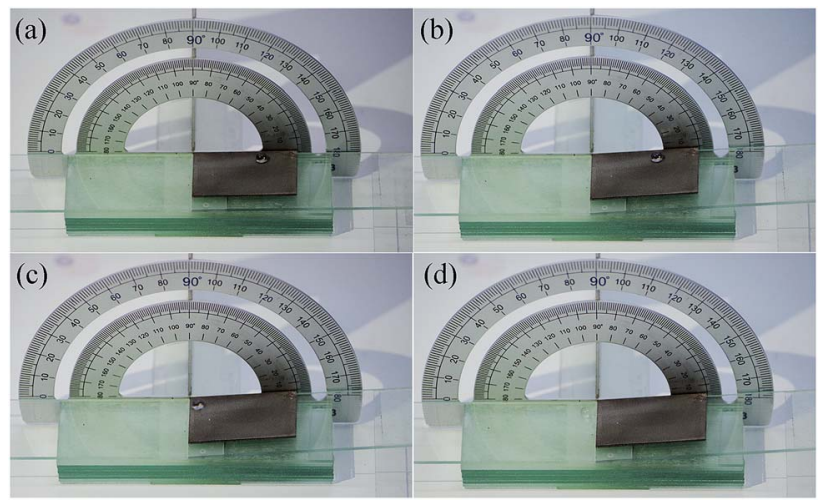

Fig. 7 Photographs of measuring the WSA of the amphiphobic surface.
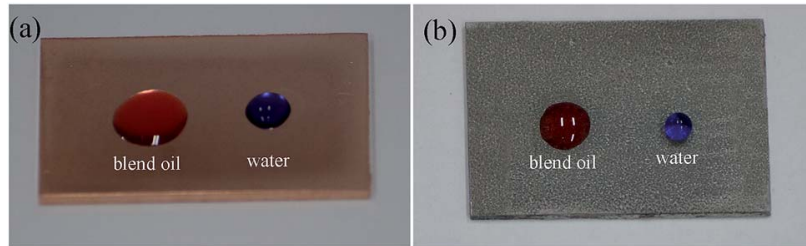

(c)

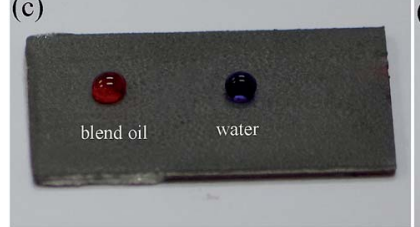

(d)

8 Different liquid droplets on (a) polished $\mathrm{Cu}$ substrate, (b) STA@AgaCu surface, (c) PFOA@AgaCu surface, (d) STA\&PFOA@Ag@Cu surface.

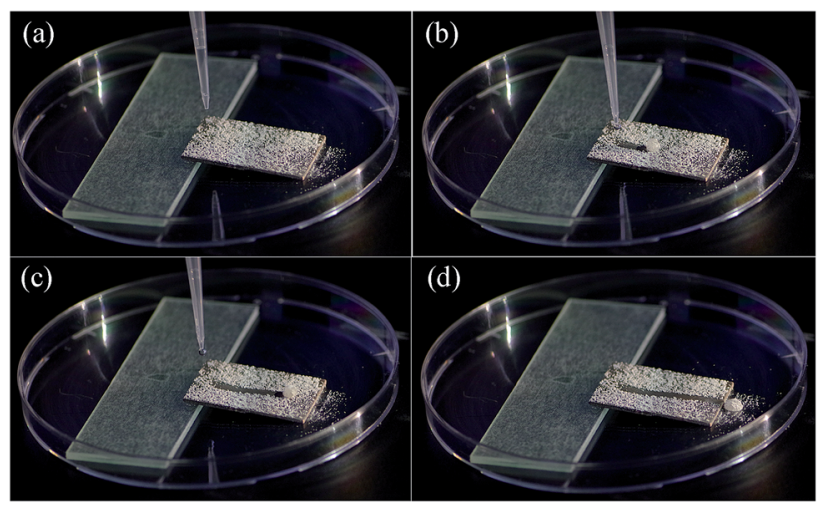

Fig. 9 Self-cleaning process of the amphiphobic surface (a) before, (b and c) during and (d) after water droplets cleaning.

PFOA content was $60 \%$, which were the optimal experimental parameters. This indicated that they were super repellent to water and highly repellent to blend oil, exhibiting the typical characteristics of amphiphobicity. ${ }^{36}$

Images of the droplets on substrates treated with different conditions are displayed in Fig. 8. Water droplets and blend oil droplets were dyed with methyl blue and Sudan red, respectively. The blend oil droplets spread over the substrate when droplets were dropped onto the polished $\mathrm{Cu}$ substrate, which indicated that the polished $\mathrm{Cu}$ substrate was hydrophilic and oleophilic (Fig. 8a). The water droplets appeared spherical on the STA@Ag@Cu surface, while the blend oil droplets spread on the surface, which indicated that the STA@Ag@Cu surface was hydrophobic and oleophilic (Fig. 8b). The PFOA@Ag@Cu surface could support the water droplets and the blend oil droplets, which proved that the PFOA@Ag@Cu surface was hydrophobic and oleophobic (Fig. 8c). However, the greater adhesion of the water droplets on the surface made the PFOA@Ag@Cu surface incapable of achieving superhydrophobicity (Video S2 $\dagger$ ). The water droplets and blend oil droplets could stand on the STA\&PFOA@Ag@Cu surface (Fig. 8d). When compared with the PFOA@Ag@Cu surface, the WSA of the STA\&PFOA@Ag@Cu surface was quite small, which further demonstrated that the surface was an amphiphobic surface with superhydrophobicity and oleophobicity. Furthermore, the STA\&PFOA@Ag@Cu surface also exhibited excellent repellency to tea droplets and milk droplets that present certain lyophobic properties.

\section{Self-cleaning property of the amphiphobic surface}

The outstanding self-cleaning property of the amphiphobic surface can protect the substrate from contamination and serve to protect the material or surface from corrosive reagents. ${ }^{6}$ Based on this, we tested the self-cleaning property of the amphiphobic surface. Fig. 9 shows the process of self-cleaning. First, the amphiphobic surface with a small angle of inclination was evenly smeared by a layer of white chalk power (Fig. 9a). After that, once the water droplets were dropped onto the surface, the white chalk power was removed immediately as the water droplets rolled off (Fig. 9b and c), owing to the small WSA of the amphiphobic surface. A clear cleaned trajectory was formed on the surface without leaving any trace of the chalk residue (Fig. 9d), which testified that the surface had an excellent self-cleaning property. The entire process was recorded in Video S3. $\dagger$

\section{Conclusions}

We proposed a facile and time-saving modification method using a mixed modified solution of stearic acid and perfluorooctanoic acid of fabricating amphiphobic surfaces on copper substrates. The effects of reaction time on surface morphology and wettability as well as the content of PFOA in the mixed modified solution on wettability were investigated. The results revealed that when the reaction time was $15 \mathrm{~min}$ and the PFOA content in the mixed modified solution was $60 \%$, the as-fabricated surfaces displayed optimal amphiphobicity with high oil contact angles of $133.5^{\circ}$, higher water contact angles of $156.8^{\circ}$ and ultra-low water sliding angles of $3^{\circ}$, which suggested superhydrophobicity and highly oleophobicity. Furthermore, the amphiphobic surface possessed an outstanding selfcleaning property, which enabled the rolling water droplets to 
remove chalk powder without any traces being left behind. The facile, easy to control, and efficient method can provide new insights into fabricating amphiphobic surfaces and can open up a new way for the basic research and practical application of amphiphobic surfaces.

\section{Conflicts of interest}

There are no conflicts to declare.

\section{Acknowledgements}

This work was supported by the Taishan Scholar Project of Shandong Province (No. TSHW20130956) and Natural Science Foundation of Shandong Province, China (No. ZR2017MA013).

\section{Notes and references}

1 T. C. Rangel, A. F. Michels, F. Horowitz and D. E. Weibel, Langmuir, 2015, 31, 3465-3472.

2 B. Qian and Z. shen, Langmuir, 2005, 21, 9007-9009.

3 C. Wang, T. Yao, J. Wu, C. Ma, Z. Fan, Z. Wang, Y. Cheng, Q. Lin and B. Yang, ACS Appl. Mater. Interfaces, 2009, 1, 2613-2617.

4 J. Li, X. Liu, Y. Ye, H. Zhou and J. Chen, J. Phys. Chem. C, 2011, 115, 4726-4729.

5 J. Tan, J. J. Hao, Z. Q. An and C. S. Liu, RSC Adv., 2017, 7, 26145-26152.

6 F. Su and K. Yao, ACS Appl. Mater. Interfaces, 2014, 6, 87628770 .

7 P. Wang, D. Zhang, R. Qiu, Y. Wan and J. Wu, Corros. Sci., 2014, 80, 366-373.

8 Y. Huang, D. K. Sarkar and X.-G. Chen, Mater. Lett., 2010, 64, 2722-2724.

9 J. Long, P. Fan, D. Gong, D. Jiang, H. Zhang, L. Li and M. Zhong, ACS Appl. Mater. Interfaces, 2015, 7, 9858-9865.

10 Z. Mao, W. Cao, J. Hu and L. Jiang, RSC Adv., 2017, 7, 4964949654.

11 H.-J. Butt, C. Semprebon, P. Papadopoulos, D. Vollmer, M. Brinkmann and M. Ciccotti, Soft Matter, 2013, 9, 418-428.

12 X. Zhang, W. Z. Zhu and I. P. Parkin, RSC Adv., 2017, 7, 3136.

13 N. J. Shirtcliffe, G. McHale, M. I. Newton and C. C. Perry, Langmuir, 2005, 21, 937-943.

14 B. J. Zhang, J. Park, K. J. Kim and H. Yoon, Bioinspiration Biomim., 2012, 7, 036011.

15 P. Ragesh, V. Anand Ganesh, S. V. Nair and A. S. Nair, J. Mater. Chem. A, 2014, 2, 14773-14797.

16 D. S. Facio, L. A. M. Carrascosa and M. J. Mosquera, Nanotechnology, 2017, 28, 265601.
17 X. Lu, Y. Peng, L. Ge, R. Lin, Z. Zhu and S. Liu, J. Membr. Sci., 2016, 505, 61-69.

18 S. Nagappan, S. S. Park, E. J. Yu, H. J. Cho, J. J. Park, W.-K. Lee and C.-S. Ha, J. Mater. Chem. A, 2013, 1, 1214412153.

19 H. Wang, D. Gao, Y. Meng, H. Wang, E. Wang and Y. Zhu, Prog. Org. Coat., 2015, 82, 74-80.

20 N. Xu, D. K. Sarkar, X. G. Chen and H. Zhang, RSC Adv., 2016, 6, 35466-35478.

21 L. Yin, J. Yang, Y. Tang, L. Chen, C. Liu, H. Tang and C. Li, Appl. Surf. Sci., 2014, 316, 259-263.

22 W. Guo, Q. Zhang, H. Xiao, J. Xu, Q. Li, X. Pan and Z. Huang, Appl. Surf. Sci., 2014, 314, 408-414.

23 J.-Y. Lee, J. Han, J. Lee, S. Ji and J.-S. Yeo, Nanoscale Res. Lett., 2015, 10, 505.

24 J. Tsibouklis and T. G. Nevell, Adv. Mater., 2003, 15, 647-650.

25 M. Xi, J. Yong, F. Chen and Q. Yang, RSC Adv., 2019, 9, 66506657.

26 H. Li, S. Yu, X. Han, E. Liu and Y. Zhao, Colloids Surf., A, 2015, 469, 271-278.

27 W. Jiang, C. Yang, Y. Zhu, Y. Liu, H. Lu and B. Liang, Appl. Surf. Sci., 2018, 457, 456-467.

28 C. Cao and J. Cheng, Surf. Coat. Technol., 2018, 349, 296-302.

29 M. Nosonovsky and B. Bhushan, Ultramicroscopy, 2007, 107, 969-979.

30 D. K. Sarkar and N. Saleema, Surf. Coat. Technol., 2010, 204, 2483-2486.

31 C. Cao and J. Cheng, Chem. Eng. J., 2018, 347, 585-594.

32 F. Chen, J. Song, Y. Lu, S. Huang, X. Liu, J. Sun, C. J. Carmalt, I. P. Parkin and W. Xu, J. Mater. Chem. A, 2015, 3, 2099921008.

33 P. Xu, F. Wang, C. Yang, J. Ou, W. Li and A. Amirfazli, Surf. Coat. Technol., 2016, 294, 47-53.

34 Y. Song, Y. Liu, B. Zhan, C. Kaya, T. Stegmaier, Z. Han and L. Ren, J. Bionic. Eng., 2017, 14, 497-505.

35 M. Xiang, M. Jiang, Y. Zhang, Y. Liu, F. Shen, G. Yang, Y. He, L. Wang, X. Zhang and S. Deng, Appl. Surf. Sci., 2018, 434, 1015-1020.

36 W. Yao, L. Li, O. L. Li, Y.-W. Cho, M.-Y. Jeong and Y.-R. Cho, Chem. Eng. J., 2018, 352, 173-181.

37 H. Li, S. Yu, X. Han, S. Zhang and Y. Zhao, J. Bionic. Eng., 2016, 13, 622-630.

38 M. Khosravi and S. Azizian, Sep. Purif. Technol., 2017, 172, 366-373.

39 G. R. T. Suyambulingam, K. Jeyasubramanian, V. K. Mariappan, P. Veluswamy, H. Ikeda and K. Krishnamoorthy, Chem. Eng. J., 2017, 320, 468-477. 